\title{
Long-Term Effects of an HIV/STI Sexual Risk Reduction Intervention for Heterosexual Couples
}

\author{
Nabila El-Bassel, ${ }^{1,3}$ Susan S. Witte, ${ }^{1}$ Louisa Gilbert, ${ }^{1}$ Elwin Wu, ${ }^{1}$ Mingway Chang, ${ }^{1}$ \\ Jennifer Hill, ${ }^{1}$ and Peter Steinglass ${ }^{2}$
}

\begin{abstract}
Received August 7, 2003; revised February 12, 2004; accepted March 22, 2004
This randomized clinical trial examined the relative efficacy of a relationship-based HIV/STI prevention intervention for women and their regular male sexual partners at 12 months postintervention. A total of 217 couples were randomized to (1) a six-session intervention provided to the woman and her sexual partner together $(n=81)$; (2) the same intervention provided to the woman alone ( $n=73)$; or (3) a one-session health information education "control" provided to the woman alone $(n=63)$. Findings suggest the intervention was efficacious in reducing unprotected sex at 12 months post-intervention, compared with the education control group. No significant differences were observed when comparing whether couples received the intervention together or when the woman received it alone.
\end{abstract}

KEY WORDS: HIV intervention; couples; sexual risk; clinical trial.

\section{INTRODUCTION}

The rate of heterosexually acquired HIV continues to rise, particularly among African American and Latina women (Centers for Disease Control and Prevention, 2001). Traditional individual or groupbased programs often fail to demonstrate increased condom use, particularly among women in long-term intimate relationships (Bryan et al., 1996; Ickovics and Yoshikawa, 1998; Schilling et al., 1995). To address these shortcomings, couple-oriented HIV/sexually transmitted infection (STI) prevention intervention models have been developed and evaluated (Allen et al., 1992; Deschamps et al., 1991; Ehrhardt and Exner, 2000; El-Bassel et al., 2003; Harvey, 2000; Higgins et al., 1991; Musaba et al., 1998; Padian et al., 1993; Voluntary HIV-1 Counseling and Testing Efficacy Study Group, 2000; Wingood and DiClemente,

\footnotetext{
${ }^{1}$ Social Intervention Group, Columbia University School of Social Work, New York, New York, USA.

${ }^{2}$ Ackerman Institute for the Family, New York, NY, USA.

${ }^{3}$ Correspondence should be directed to Nabila El-Bassel, Social Intervention Group, Columbia University School of Social Work, 125S Amsterdam Avenue, New York, NY 10027, USA; e-mail: ne5@columbia.edu.
}

2000). Relationship-based approaches to HIV/STI prevention may better address the context of gender and power in relationships and facilitate the development of couple communication skills that enable long-term intimate partners to negotiate condom use (El-Bassel et al., 2003; El-Bassel et al., 2001; Kalichman et al., 1993; Misovich et al., 1997; O'Leary, 2000).

Traditional couple counseling is a modality where partners receive treatment conjointly. The key difference between couple counseling, as opposed to individual or group counseling, is that the couple, rather than the individual, is the target of change. Literature on couple-based therapy also suggests that relationship-based interventions can be provided either to one partner alone or to the couple together (Weiner-Davis, 1998). However, relationship-based interventions delivered to the couple together may be more effective for several reasons. First, research suggests that individuals acting unilaterally to introduce safer sex practices may be confronted with negative reactions including isolation, threats to terminate the relationship, or physical violence (Harlow et al., 1993; Kalichman et al., 1998; Wingood and DiClemente, 1998). Second, the expectation that an 
individual participating alone in an intervention can convey new knowledge and skills to a sexual partner assumes $\mathrm{s} / \mathrm{he}$ have the requisite relationship-specific communication skills. Third, the supportive environment of couple counseling may enable intimate partners to feel safer disclosing highly personal information (e.g., extra-dyadic relationships, STI histories) to their partners that will enable them to gain a more realistic appraisal of their risks for HIV/STI transmission as a couple (Remien, 1997).

A number of studies have demonstrated that providing intervention sessions jointly to both members of a dyad is efficacious in promoting HIV counseling and testing, as well as in increasing condom use (Allen et al., 1992; Deschamps et al., 1991; Higgins et al.,1991; Musaba et al., 1998; Padian et al.,1993; Voluntary HIV-1 Counseling and Testing Efficacy Study Group, 2000). Most of these studies, however, were conducted outside of the United States and targeted HIV mixed status couples, where one partner is HIV negative and the other is HIV positive.

Allen et al. (1992) recruited 60 HIV mixed status couples in Rwanda to determine whether HIV testing and counseling increased condom use and decreased heterosexual HIV transmission. The proportion of these couples using condoms after receiving counseling and testing increased from 4 to $57 \%$ at one-year follow-up. At two-year follow-up, two of the 23 HIV negative men and six of the 30 HIV negative women had seroconverted. This seroconversion rate among women was less than half of that estimated for similar women in mixed HIV status couples who had not received counseling. Findings indicate that counseling and testing for couples were associated with a substantial increase in condom use and a lower rate of new HIV infections. Padian et al. (1993) recruited 144 HIV mixed status couples from various HIV counseling and testing sites throughout California and provided couple counseling and risk assessments every 6 months. Both condom use and sexual abstinence increased over time, but the greatest behavioral change occurred between intake and the first follow-up visit.

In a study testing the effect of female condom use on unprotected sexual acts, Musaba et al. (1998) recruited 99 heterosexual couples from a Zambian hospital clinic where one partner was symptomatic for an STI. All couples were provided with female and male condoms and spermicides, and were counseled to use either type of condom plus a spermicide for each act of intercourse. Overall, findings demonstrated that there were significant reductions in the proportion of unprotected sexual acts at 3,6 and 12-month assessments.

The Voluntary HIV-1 Counseling and Testing Efficacy Study Group (2000) randomly assigned 586 couples, recruited through local media and direct solicitation in Kenya, Tanzania and Trinidad, to receive voluntary counseling and testing services (VCT) or basic health information. Couples assigned to VCT reduced unprotected intercourse with their study partners significantly more than those assigned to health information.

One key question regarding the ultimate utility of any prevention intervention is whether the program can sustain risk reduction behavior change over longer periods of time. Published studies on the maintenance of sexual risk reduction behavioral change, or reports on long-term outcomes of studies, are increasing. Of 15 published studies we identified with follow-up assessment periods of approximately 1 year or more, 9 demonstrated maintenance of behavior change (Ehrhardt et al., 1992; El-Bassel and Schilling, 1992; Voluntary HIV-1 Counseling and Testing Efficacy Study Group, 2000; Kamb et al., 1998; Landis et al., 1992; McCusker et al., 1993; NIMH Multisite HIV Prevention Trial, 1998; Padian et al., 1993; Shain et al., 1999). Only two of these tested the long-term efficacy of a couple-level intervention (Padian et al., 1993).

Project Connect tested the efficacy of a relationship-based intervention on increasing condom use among 217 low income, predominantly minority women and their regular sexual partners, who were at elevated risk for HIV/STIs. This paper examines the primary outcomes of Project Connect at 12 months post-intervention. The objectives of this study were to examine: (1) whether a sixsession relationship-based intervention to prevent HIV/STIs would be efficacious in increasing the proportion of protected sexual acts and decreasing the number of unprotected sexual acts at 12 month follow up, compared to a control condition consisting of a single session of HIV/STI education, and (2) whether the intervention would be equally efficacious when the woman and her partner received the relationship-based intervention together compared with when the woman received the relationship-based intervention alone. Findings from the women's and their male study partners' reports from the 3-month follow-up are published elsewhere (El-Bassel et al., 2003). Findings from 3 -month outcomes suggest the intervention was efficacious in reducing unprotected sex and increasing 
the proportion of protected sex acts. No significant differences in outcomes were observed when comparing couples who received the intervention together with woman who received the intervention alone. Unfortunately, funding was not allocated to interview men at 12 months post-intervention; therefore, study data in this paper are from women only. The findings of this paper provide new knowledge on behavioral change over time.

\section{METHODS}

The study was conducted between 1997 and 2001. Women were recruited from hospital-based outpatient clinics in Bronx, New York. Bilingual Spanish/English-speaking recruiters approached women in waiting rooms and handed them study flyers. Those who agreed to participate in the study completed a 10-min, face-to-face eligibility-screening interview. Women who met eligibility criteria were asked to recruit their regular male sexual partners. In order to gain their partners' cooperation, the women were given a letter that could be shared with their partners describing the project and its purpose. A woman was eligible for the study if she: (1) was between 18 and 55 years old; (2) had a regular, male sexual partner whom she identified as a boyfriend, spouse, or lover; (3) was in a long-term relationship, operationalized as involvement with this partner for the past 6 months and intent to stay with him for at least 1 year; (4) had at least one episode of unprotected vaginal or anal sex with this partner in the past 30 days; (5) did not report any severe physical or sexual abuse by this partner within the past 6 months, according to subscales on sexual coercion from the Revised Conflict Tactics Scales (Straus et al., 1996); and (6) was a patient at one of the hospital's outpatient clinics. To be eligible, a woman also had to know or suspect that her partner met at least one of the following HIV/STI risk criteria: (1) he had sex with other women or men in the past 90 days; (2) he had been diagnosed with or exhibited symptoms of an STI in the past 90 days; (3) he had injected drugs in the past 90 days; and/or (4) he is HIV positive. Finally, enrollment in the study required participation of the woman's main, male sexual partner. Prior to the baseline interview, the female and male partners completed written, informed consent procedures. Women were eligible for the study regardless of prior or recent participation in other HIV prevention studies. The study protocol was reviewed and approved annually by the Institutional Review Boards at the study site and research institution.

Of the 2,416 women screened for the study, 388 women met the eligibility criteria. Of the eligible women, 217 agreed to participate. 52 of the eligible women were unable to enroll due to partner refusal. Both eligibility and enrollment rates are consistent with other major HIV prevention trials (NIMH Multisite HIV Prevention Trial, 1998). Eligible women who participated and eligible women who did not enroll were similar in age, education, marital status, and HIV risk behaviors (Wu et al., 2001). At baseline, simultaneous, but separate, interviews with gender-matched interviewers took place with each partner. Couples were then randomly assigned to one of three study conditions (Fig. 1): (1) the couple condition (couples), six weekly relationship-based sessions, in which both a woman and her partner received the intervention; (2) the woman-alone condition (womanalone), in which only the woman received the same intervention; or (3) the education control condition (education), in which the woman alone received one HIV/STI information session. All participants were asked to return for follow-up assessment interviews three months after the single education session or the final couple or woman-alone intervention session. Due to funding shortages noted, only women were asked to return for follow-up assessment at 12 months post-intervention.

\section{MEASURES}

Socio-demographic variables (e.g., age, ethnicity, homelessness, length of relationship and pregnancy intention) and risk characteristics (e.g., HIV status and frequency of non-injection drug use) were determined by collecting demographic data at baseline from both women and their partners. Non-injection drug use was assessed in terms of the frequency of drug use during the previous 90 days (crack, cocaine, heroin).

Relationship variables defined here are pretreatment variables that were used to examine similarity between the comparison groups in the propensity score matching (See Data Analysis). Financial dependency was defined by a single item assessing to what extent a woman was financially dependent on her current partner. Relationship Satisfaction was assessed using the Relationship Assessment Scale (RAS), a 7-item measure of global relationship satisfaction (Cronbach's alpha $=.86)$ (Hendrick et al., 1998). Physical and/or 


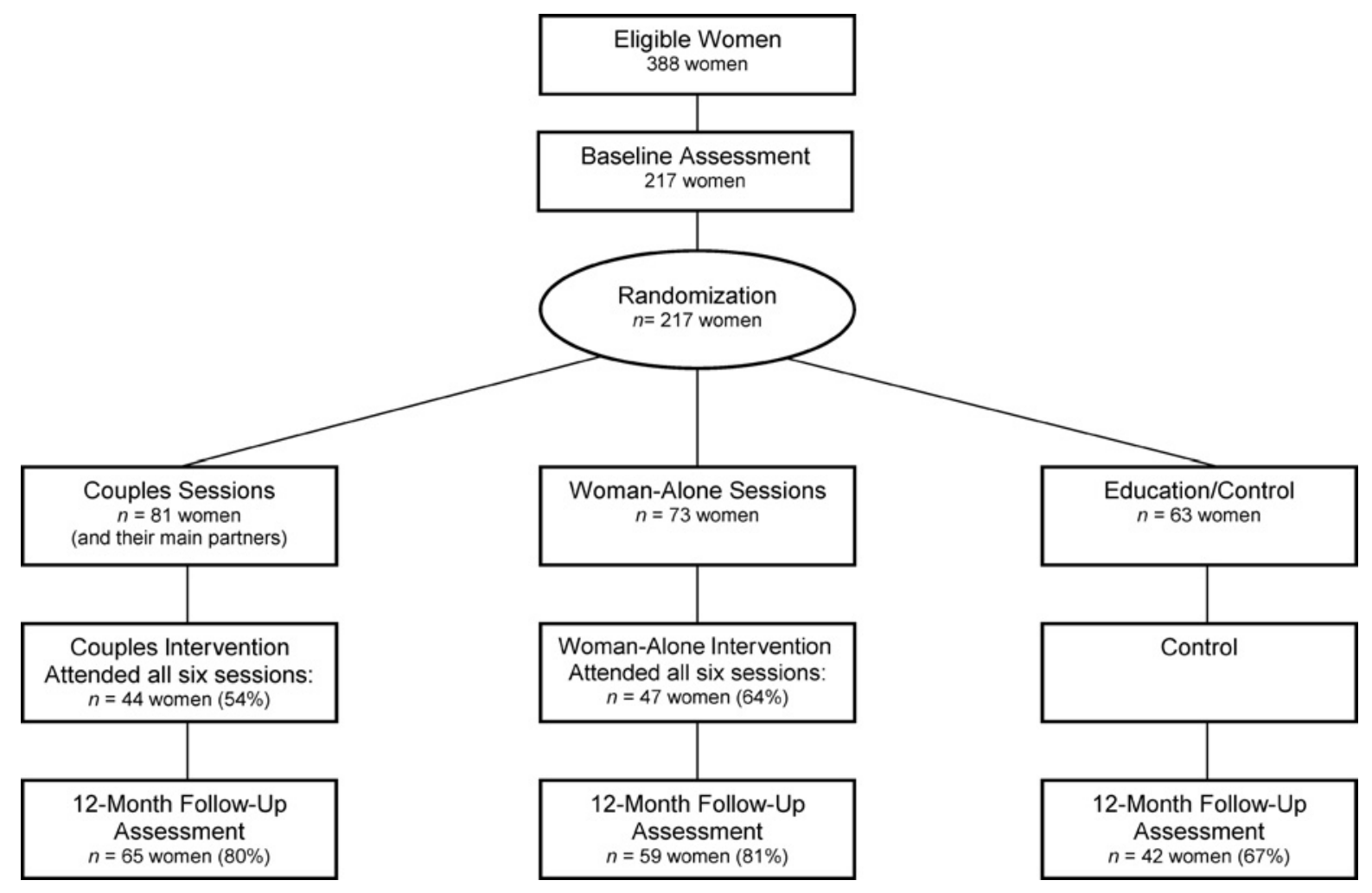

Fig. 1. Design of Project Connect.

sexual abuse by the study partner was measured using Physical Assault and Sexual Coercion Scales from the Revised Conflict Tactics Scales (CTS2) (Cronbach's alphas .86 and .87, respectively) (Straus et al., 1996). The CTS2 assesses prevalence, severity and frequency of abusive behaviors by the study partner in the past 6 months and lifetime. Condom Negotiation Self-Efficacy, defined as one's perceived ability to protect oneself during sex and to negotiate safer sex, was assessed using a 20-item scale (Cronbach's alpha $=.86$ ) (Marin et al., 1997). Sexual comfort, defined as comfort with sexual issues and one's own sexuality, was measured using a 7-item, 4-point Likert scale ranging from (1) very uncomfortable to $(4)$ comfortable (Cronbach's alpha $=.85$ ) (Marin et al., 1997). Sexual Communication, defined as the level of comfort talking to one's partner about sex, condoms, condom use, sexual desires or fantasies, and what feels most pleasurable sexually to him or her, was measured using an 10-item, 5-point Likert scale ranging from very uncomfortable (1) to very comfortable $(5)$ (Cronbach's alpha $=.81)$. This measure was adapted from the Sexual Comfort Scale (Marin et al., 1997). Relationship Problem Solving was assessed using the Marital Problem Solving Scale (MPSS). The MPSS is a 9-item measure, using a 7-point Likert scale, which has been used extensively to examine problem-solving abilities within marital dyad members (Cronbach's alpha $=.95)$ (Baugh et al., 1982).

Three primary outcomes were assessed for the past 90 days: (1) number of unprotected acts of vaginal intercourse with the study partner, (2) proportion of protected acts of vaginal intercourse with the study partner, and (3) always used a condom during sex, or $100 \%$ condom use (Gilbert et al., 2000). Each participant was asked the following questions: "How many times did you engage in vaginal sex with your study partner in the past 90 days;" "Of these times, how many times did you use a male condom during vaginal sex with your study partner in the past 90 days;" and "Of these times, how many times did you use a female condom during vaginal sex with your study partner." The proportion of protected acts of vaginal intercourse was calculated by dividing the sum of male and female-condom protected acts by the total number of vaginal sex acts. The total number of unprotected acts was calculated by taking the total number of vaginal sex acts and subtracting the sum of male and 
female-condom protected acts. One hundred percent condom use was indicated when the sum of protected acts was equal to the total number of vaginal sex acts. Although we also measured STI diagnosis in the past 90 days, it was not used as an outcome because of the low number of participants who reported being diagnosed with an STI during 90 days prior to the 12-month follow-up ( $n=8$, less than $5 \%$ of observed cases).

Whether provided conjointly to partners or oneon-one with the woman, the content of each intervention session was the same, incorporating concepts from the AIDS Risk Reduction Model (ARRM) (Catania et al., 1990), the ecological perspective (Bronfenbrenner, 1979), previous related empirical research on HIV/STI prevention with couples (NIMH Multisite HIV Prevention Trial, 1998), and qualitative pilot data summarized from local community consultants interviewed during the developmental phase of the study (El-Bassel et al., 2001; Sormanti et al., 2001).

The ARRM is a conceptual framework to organize information and skills directed at HIV risk reduction. This eclectic model integrates constructs from the theory of reasoned action (Ajzen and Fishbein, 1980; Fishbein and Middlestadt, 1989), social cognitive theory (Bandura, 1986, 1994), and health-belief approaches (Becker, 1974; Janz and Becker, 1984). The ecological perspective (Bronfenbrenner, 1979; Fagan and Wexler, 1987; Moss and Tarter, 1993; Reynolds, 1993; Rojek et al., 1989) provides a way to conceptualize a contextually- and relationshipspecific approach to HIV risk reduction (Moss and Tarter, 1993). This perspective emphasizes the various levels of contextual factors, from ontogenetic (individual) to macro level (e.g., cultural and gender role norms), that play a role in establishing and maintaining protective behaviors.

The intervention consisted of six relationshipbased HIV/STI prevention sessions (El-Bassel et al., 2001). Intervention sessions met once a week, lasted $2 \mathrm{hrs}$, and began either the day of baseline or on a day scheduled within the following 2 weeks. Each participant received an incentive of $\$ 20$ for each session completed. Intervention sessions were generally completed within 7 weeks of enrollment. Couple sessions were rescheduled if one partner was absent. Details about the intervention are published elsewhere (El-Bassel et al., 2001). Briefly, the intervention sessions for both couples and woman-alone centered on the woman and her recruited partner, with a strong emphasis on the relationship context. Sessions addressed issues of intimacy and closeness in the relationship, as well as the meaning of monogamy and trust, and explored how these factors act as barriers to HIV/STI protection among couples. The intervention emphasized the importance of relationship communication, negotiation, and problem-solving skills, and highlighted how relationship dynamics may be affected by gender roles and expectations. The session content emphasized each couple's contribution to enhancing the future health of ethnic communities hardest hit by HIV/AIDS.

The single education control session lasted $1 \mathrm{hr}$ and was provided immediately following baseline and randomization. Content was standardized using a video-based format with a brief question and answer period following.

All facilitators were female. Facilitator requirements included (1) having a Master's Degree in Counseling, Social Work or another helping profession or being a second year Master of Social Work student; (2) having had 2 or more years experience in HIV prevention or a related field; and (3) successfully completing an intensive 5 day facilitator training course.

Of those assigned to "couples," $100 \%$ attended the first orientation session and $54 \%$ attended all intervention sessions. Among those assigned to "woman alone," $100 \%$ attended the first orientation session and at least one session, and $64 \%$ attended all intervention sessions (see Fig. 1).

To ensure the quality and consistency of interventions, facilitators received a standardized training, used structured intervention protocols, met on a weekly basis with a clinical and task supervisor, and had routine monitoring (via audiotape) and feedback by an on-site supervisor (a random 10\% of sessions were monitored). In addition, process evaluations assessing intervention content and client satisfaction with the interventions were conducted by surveying participants in the final session (Schiff et al., 2003).

\section{DATA ANALYSIS}

Quantitative estimates of treatment effects at 12-month follow-up relied on ordinary least squares (OLS) regression for the proportion of protected sex, logistic regression for the binary outcome variable of $100 \%$ condom use, and OLS regression with the number of unprotected acts of intercourse in the prior 90 days transformed by adding one and 
then taking the natural logarithm to obtain a more normally distributed variable. In all regression models, we adjusted for the pre-treatment variables of HIV status, intention to get pregnant, and the baseline of the outcome variable of interest to allow for more efficient estimation of the treatment effects.

We used two different coding schemes to examine treatment effects where the three-armed design implemented the two active conditions of the same intervention, but differed in whether the women received the intervention alone or with her regular partner. In the first scheme, two contrast codes were created to directly test the study's two primary aims. The first contrast code allows for the direct test of the first aim-whether a six-session HIV/STI relationship-based intervention would be efficacious compared to one education control session (i.e., couple and woman-alone vs. education); the second contrast code allows for the examination of the second aim - whether the intervention would be more efficacious when the woman and her partner received the HIV/STI relationship-based intervention together or when the woman received it alone (i.e., couple vs. woman-alone). The second scheme relied on dummy coding, allowing for straightforward pairwise comparisons of the relative efficacies of (1) couple vs. education; and (2) woman alone vs. education.

An intention-to-treat (ITT) approach to estimating treatment effects requires that all randomized cases be included in the analyses, including those who were unavailable or did not return for the 12-month follow-up assessment. In our study, follow-up rates differed for each arm: 80,81 , and $67 \%$ for couples, woman-alone, and education respectively. Attrition analyses examining differences between those who returned for the 12-month follow-up assessment and those who did not, with respect to variables measured at baseline, indicated only one significant difference: among those assigned to the woman-alone condition, those who had completed high school or at least obtained a GED were significantly more likely to attend the 12-month follow-up compared to those who had less than a high school diploma. We employed multiple imputation procedures (MI) (Rubin, 1987) to impute values for missing data for all variables, including co-variates and outcome variables. Briefly, MI uses the information that is observed or measured for a participant in order to predict values for variables where that individual's information is missing. MI replaces each missing value with several imputed values.
Taken together, MI generates more valid standard errors compared to ad hoc and single imputation methods such as last-observation carried forward, worst case scenario, replacement with mean, etc. The only exception was for five women that died before the 12-month follow-up assessment and who were excluded from the analyses.

An ITT approach prevents potential bias in treatment effect estimates stemming from differential adherence or attrition. However, ITT analyses may also mask potentially interesting and important treatment effects by classifying those who adhered to treatment protocols the same as those who did not adhere well to their assigned treatment. Therefore, we also examined the treatment effects using the subset of "completers," (i.e., those assigned to couples or to womanalone who attended all six sessions and thus received a "complete dose" of the intervention). Since attendance is technically a post-treatment variable-that is the likelihood of attending all six sessions may be differentially affected by the differences in intervention and/or modality - we employed propensity score matching to identify "matches" (those similar in terms of measured pre-treatment variables) for the completers from among those in the education arm. Since we expect the completers to be different from those who don't complete the full regimen in terms of their pre-treatment characteristics, comparisons between completers and all the participants in the education condition are likely to produce biased estimates because they destroy the initial randomization. (Dehejia and Sadek, 1999; Rosenbaum and Rubin, 1983, 1985). Pre-treatment variables included age, ethnicity, homelessness, financial dependency, length of relationship, HIV status, injection drug use, noninjection drug use, physical and/or sexual abuse by the study partner, safer sex communication scale, sexual comfort scale, condom use negotiation self-efficacy, relationship problem-solving and relationship satisfaction. These variables were selected based on theoretical considerations guided by AIDS Risk Reduction Model (Catania et al., 1990) and the ecological perspective (Bronfenbrenner, 1979). After propensity score matching, various diagnostics, including robust approaches such as KolmogorovSmirnov and Shapiro-Wilk tests, were employed to verify that matched groups did not differ significantly from each other with respect to pre-treatment attributes. After verification of appropriate matching, treatment effect estimates for completers were calculated using the same regression procedures described above. 
Table I. Socio-Demographic and Risk Behavior Characteristics for the Baseline Participants Randomized in the Study and for High-Dose Participants

\begin{tabular}{|c|c|c|c|c|c|}
\hline & \multicolumn{3}{|c|}{ As randomized (ITT) } & \multicolumn{2}{|c|}{ Completers } \\
\hline & $\begin{array}{l}\text { Couple } \\
(n=81)\end{array}$ & $\begin{array}{l}\text { Woman alone } \\
\quad(n=73)\end{array}$ & $\begin{array}{c}\text { Education } \\
(n=63)\end{array}$ & $\begin{array}{l}\text { Couple } \\
(n=44)\end{array}$ & $\begin{array}{l}\text { Woman alone } \\
\quad(n=47)\end{array}$ \\
\hline \multicolumn{6}{|l|}{ Socio-Demographics (\%) } \\
\hline$<25$ years of age & 9.9 & 8.2 & 9.5 & 6.8 & 8.5 \\
\hline African American & 54.3 & 54.8 & 54.0 & 47.7 & 63.8 \\
\hline Hispanic & 38.3 & 43.8 & 36.5 & 40.9 & 34.0 \\
\hline$\geq$ High school or GED & 42.0 & 37.0 & 55.6 & 45.5 & 48.9 \\
\hline Never married & 67.9 & 57.5 & 52.4 & 65.9 & 53.2 \\
\hline Employed & 11.1 & 15.1 & 17.5 & 9.1 & 17.0 \\
\hline Income $<\$ 5,000 /$ year & 64.2 & 72.6 & 68.3 & 65.9 & 72.3 \\
\hline \multicolumn{6}{|l|}{ Risk (\%) } \\
\hline$>1$ partner (past 90 days) & 24.7 & 23.3 & 14.3 & 22.7 & 29.8 \\
\hline Ever HIV tested & 95.1 & 91.8 & 92.1 & 95.5 & 89.4 \\
\hline HIV positive & 25.9 & 21.9 & 15.9 & 22.7 & 19.1 \\
\hline HIV unknown & 6.2 & 11.0 & 7.9 & 6.8 & 12.8 \\
\hline $0 \%$ condom use (past 90 days) & 64.2 & 71.2 & 76.2 & 70.5 & 76.6 \\
\hline $100 \%$ condom use (past 90 days) & 0 & 2.7 & 3.2 & 0 & 0 \\
\hline Non-injected drugs (past 90 days) & $60 .^{a}$ & $63.8^{a}$ & $44.6^{a}$ & $57.1^{b}$ & $63.6^{b}$ \\
\hline Injected drugs (past 90 days) & 11.1 & 5.5 & 6.3 & 6.8 & 6.4 \\
\hline \multicolumn{6}{|l|}{ Mean } \\
\hline Number of unprotected sex acts & 27.7 & 24.2 & 23.5 & 29.9 & 21.1 \\
\hline Proportion of protected sex acts & .184 & .125 & .118 & .154 & .093 \\
\hline
\end{tabular}

${ }^{a}$ Sample sizes are 79,69 , and 56 for couples, women alone and education, respectively.

${ }^{b}$ Sample sizes are 42 and 44 for couples and women alone, respectively.

\section{RESULTS}

\section{Socio-Demographic and Risk Characteristics}

Table I summarizes the participants' characteristics measured at baseline and presented by treatment assignment (columns under the "As randomized" heading). These findings were also used to examine the success of the randomization in balancing pre-treatment characteristics. No significant differences were observed on the pre-treatment characteristics among the different study arms.

The completers in both active intervention arms exhibited more discernable differences with respect to several socio-demographic background and sexual risk behavior variables as compared to those assigned to the education condition. In the couples condition, the completers were less likely to be under 25 years old, African American and/or employed as compared to those assigned to the educational control condition; these differences between the completers in the couple condition and the education condition sample are greater than the differences between the entire couple condition sample and the education condition sample on pre-treatment characteristics. There are also greater discrepancies between the com- pleters who were assigned to woman-alone and those assigned to education, with woman-alone completers having a greater likelihood of being African American, having more than one partner, not knowing one's own HIV status, and ever having had an STI. These discrepancies highlight the necessity of using appropriate matching procedures prior to estimating treatment effects among completers.

In an effort to illustrate the relative size of intervention effects achieved, Fig. 2 provides simple comparisons of baseline and follow-up reports on all three outcomes in each of the three study conditions.

\section{First Study Aim}

Here we examine whether a relationship-based HIV/STI intervention is more efficacious compared to a one-session HIV/STI education control condition (couple, woman-alone vs. education)

\section{ITT Approach}

In Table II, the columns labeled "Intervention" present the results for the first study aim. Effect sizes are reported using intervention assignment contrast/dummy variables coefficients from OLS 


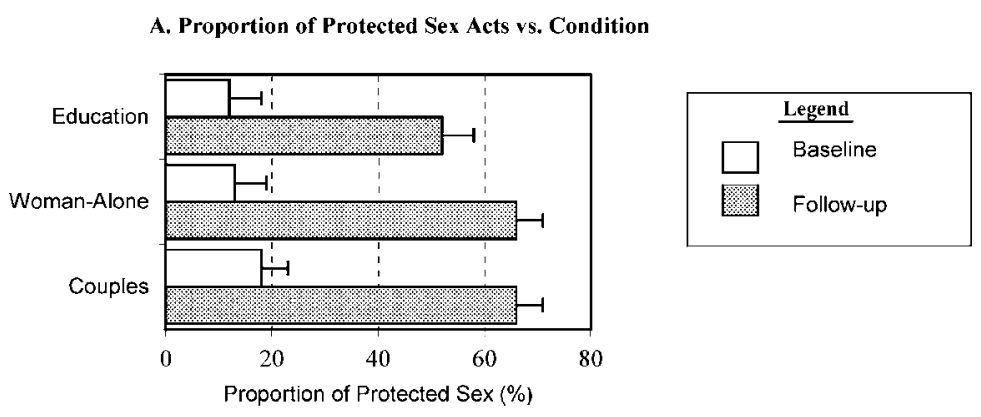

B. $100 \%$ Protected Sex Aets vs. Condition
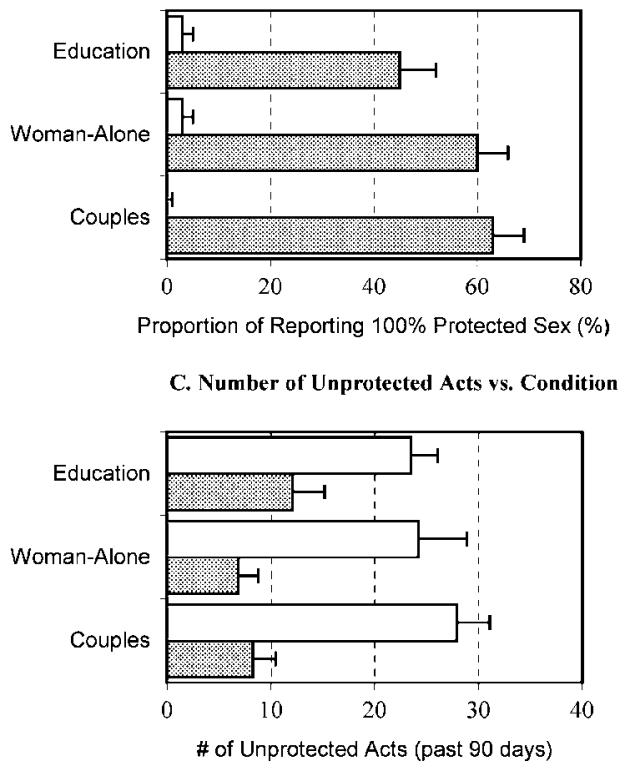

Fig. 2. Outcome variables among the different treatment assignments.

regression for proportion of protected acts and number of unprotected acts, and as odds ratios obtained from the coefficients of assignment contrast/dummy variables in logistic regressions used for $100 \%$ condom use. Using an ITT approach, all point estimates regarding the efficacy of the relationshipbased HIV/STI intervention are in the direction of reduced risk, with the number of unprotected acts of intercourse being the measure displaying the most significant beneficial effect. Women who were assigned to the active intervention arms, irrespective of whether they received the sessions alone or with their partners, were more likely than the women who received the one HIV/STI education session to increase the proportion of protected sexual acts, have $100 \%$ condom use, and to significantly decrease the number of unprotected sexual acts at 12-month follow-up assessment (see Table II).

\section{Completers (Attended All Six Sessions)}

Results from analyses comparing those assigned to the couple or woman-alone condition who attended all six sessions of the intervention to appropriately matched counterparts assigned to the education session were essentially identical to those obtained from ITT analyses for the outcome variable of number of acts of unprotected sex. For proportion of protected sex, estimates using completers appeared more beneficial $(b=.17, p=.05)$ than those obtained from ITT analyses $(b=.11, p=.12)$; a similar trend was observed for the outcome variable of $100 \%$ condom use (adjusted OR $=2.41, p=.07$ for completers vs. adjusted OR $=1.72, p=.14$ for ITT).

The results indicate that participants who attended all six sessions, irrespective of whether the women received the sessions alone or with their 
Table II. Estimates of Treatment Effects of Receiving The Relationship-Based Intervention and the Differential Effects Based on Modality Of Intervention Delivery

\begin{tabular}{|c|c|c|c|c|}
\hline & \multicolumn{2}{|c|}{ Intervention $^{a, c}$} & \multicolumn{2}{|c|}{ Modality $b, c$} \\
\hline & $\begin{array}{c}\text { As randomized } \\
(\text { ITT }) \\
(N=212)\end{array}$ & $\begin{array}{l}\text { Completers } \\
(N=133)\end{array}$ & $\begin{array}{c}\text { As randomized } \\
(\text { ITT }) \\
(N=212)\end{array}$ & $\begin{array}{c}\text { Completers } \\
(N=63)\end{array}$ \\
\hline Proportion of protected sex acts ${ }^{d}$ & $.11(.12)$ & $.17^{*}(.05)$ & $.02(.79)$ & $-.13(.24)$ \\
\hline $100 \%$ condom use $e^{e}$ & $1.72(.14)$ & $2.41 *(.07)$ & $.87(.72)$ & $.69(.61)$ \\
\hline Number of unprotected sex acts $f$ & $-.38^{* *}(.04)$ & $-.38 *(.09)$ & $.08(.76)$ & $.23(.59)$ \\
\hline
\end{tabular}

Note. All regression models included adjustment for the following covariates: HIV status, pregnancy intention, and the baseline measurement of the study outcome.

${ }^{a}$ Contrast code for (couple, woman-alone, education $)=(1 / 3,1 / 3,-2 / 3)$.

${ }^{b}$ Contrast code for (couple, woman-alone, education $)=(-1 / 2,1 / 2,0)$.

${ }^{c}$ Although these contrast codes are not orthogonal given the unequal group sizes, we present estimates using these codes since they are directly interpretable and also represent the most relevant estimates should the study be replicated. Analyses carried out using orthogonal contrast codes specific to the group sizes in this study have the same $p$-values within rounding error as those shown in this table.

${ }^{d}$ The reported numbers are OLS regression coefficients (associated $p$-values are indicated in the parentheses).

${ }^{e}$ The reported numbers are adjusted odds ratios estimated using logistic regression (associated $p$-values are indicated in the parentheses).

${ }^{f}$ The reported numbers are reverse transformed from the regression coefficient: $\exp (\mathrm{b})-1$ (associated $p$-values are indicated in the parentheses).

${ }^{*} p<.1{ }^{* *} p<.05$.

partners, were more likely to always use condoms compared to their matched counterparts in the education condition (see Table II).

\section{Second Study Aim}

Here we test whether the efficacy of the HIV/STI relationship-based intervention differs if a woman received the intervention alone compared to if she received it with her regular sexual partner (woman-alone vs. couple).

\section{ITT Approach}

The columns labeled "Modality" in Table II present results from regression analyses examining the second aim. No significant differences were found between the two study arms on the three outcomes.

\section{Completers (Attended All Six Sessions)}

Examining the same aims using only the participants who completed all six sessions versus their matched counterparts assigned to education, the findings also suggest that there were no significant differences between participants in the couple vs. the woman-alone condition (see Table II).

\section{Pair-Wise Comparison}

Here we examine whether women who were assigned to the couples arm were more likely to reduce their HIV risk behavior on the three outcomes compared to the women who were assigned to one HIV/STI education session.

\section{ITT Approach}

Estimation of treatment effects of assignment to couples versus education is presented in Table III. Using an ITT approach, estimates for the three outcomes are all in the direction of reduced HIV/STI risk, with the decrease in number of unprotected acts reaching significance at the $95 \%$ confidence level. Women assigned to the couple condition reported a greater proportion of protected sexual acts, an increased likelihood of $100 \%$ condom use, and significantly fewer unprotected sexual acts at the 12-month follow-up compared to the women who were assigned to the education session.

\section{Completers (Attended All Six Sessions)}

Efficacy estimates for the three outcomes shifted even more in a positive direction when a matching approach was used to examine treatment effects among women assigned to the couple condition who attended all six sessions compared to their matched 
Table III. Estimates of Treatment Effects Using Pair-Wise Comparisons of Each Active Intervention Arm Versus the Education Control Condition

\begin{tabular}{|c|c|c|c|c|}
\hline & \multicolumn{2}{|c|}{ Couple vs. Education $^{a}$} & \multicolumn{2}{|c|}{ Woman alone vs. Education $^{b}$} \\
\hline & $\begin{array}{c}\text { As randomized } \\
(\text { ITT }) \\
(n=212)\end{array}$ & $\begin{array}{c}\text { Completers } \\
(n=71)\end{array}$ & $\begin{array}{c}\text { As randomized } \\
(\text { ITT }) \\
(n=212)\end{array}$ & $\begin{array}{c}\text { Completers } \\
(n=70)\end{array}$ \\
\hline Proportion of protected sex acts ${ }^{c}$ & $.10(.20)$ & $.13(.31)$ & $.12(.14)$ & $.13(.28)$ \\
\hline $100 \%$ condom use ${ }^{d}$ & $1.84(.16)$ & $3.74^{* *}(.05)$ & $1.60(.23)$ & $1.88(.32)$ \\
\hline Number of unprotected sex acts ${ }^{e}$ & $-.40^{* *}(.04)$ & $-.56^{* *}(.04)$ & $-.36^{*}(.09)$ & $-.45(.17)$ \\
\hline
\end{tabular}

Note. All regression models included adjustment for the following covariates: HIV status, pregnancy intention, and the baseline measurement of the study outcome.

${ }^{a}$ Dummy code for (couple, woman-alone, education $)=(1,0,0)$.

${ }^{b}$ Dummy code for (couple, woman-alone, education $)=(0,1,0)$.

${ }^{c}$ The reported numbers are OLS regression coefficients (associated $p$-values are indicated in the parentheses).

${ }^{d}$ The reported numbers are adjusted odds ratios estimated using logistic regression (associated $p$-values are indicated in the parentheses).

${ }^{e}$ The reported numbers are reverse transformed from the regression coefficient: $\exp (\mathrm{b})-1$ (associated $p$-values are indicated in the parentheses).

${ }^{*} p<.1 ;{ }^{* *} p<.05$.

counterparts assigned to the education condition. Women who attended all six intervention sessions with their partners were significantly more likely to always use condoms and decrease the number of unprotected acts at the 12 month follow-up assessment compared to their matched counterparts assigned to the education condition (see Table III).

\section{Pair-Wise Comparison}

Here we examine whether women assigned to the woman-alone condition reduced their HIV risks using the three outcome measures compared to the women who were assigned to one HIV/STI education session.

\section{ITT Approach}

Estimates of the relative efficacy of the womanalone condition versus the education condition are also presented in Table III. Estimates for the three outcomes using an ITT approach indicate a reduction in HIV/STI risk behavior for women in the womanalone arm compared to similar counterparts assigned to the education arm (see Table III). Statistically significant differences at the $90 \%$ confidence level were observed between woman-alone and education condition for the number of unprotected sex acts (see Table III).

\section{Completers (Attended All Six Sessions)}

Comparisons between women assigned to woman-alone who attended all six sessions and matched counterparts assigned to education show slightly more beneficial treatment effects especially in reducing the number of unprotected sex estimates compared to estimates using an ITT approach (see Table III).

\section{DISCUSSION}

Even though most women living with HIV/AIDS were infected through heterosexual intercourse with regular partners, the science of couples-based HIV/STI prevention intervention in the United States is still in its early stages. This study examined whether a relationship-based HIV/STI intervention could demonstrate sustained outcome effects at 12-month follow-up, and whether the intervention was more efficacious over time when women received the intervention with their regular partners versus women who received the intervention alone. We used both an ITT approach and a standard matching approach in our analyses, measuring treatment effects on three major outcomes-proportion of unprotected sexual acts, $100 \%$ condom use, and number of unprotected sexual acts. Findings suggest that the relationship-based intervention is efficacious in reducing HIV/STIs, particularly by reducing the number of unprotected sex acts at 12 months post-intervention. Women assigned to an active intervention arm, irrespective of whether they received the sessions alone or with their partners, significantly decreased the number of unprotected sexual acts at 12 months and improved on the other two outcome measures (proportion of protected sexual acts and consistent condom usage). When those who attended all intervention sessions of the active 
conditions were compared with a matched set of participants from the education condition, findings showed that that the women who completed either of the six-session relationship-based conditions were more likely than their counterparts in the education condition to increase the proportion of protected sexual acts, decrease the number of unprotected acts (approaching significance), and increase consistent condom use. For all outcome variables, estimates using ITT and matching approaches indicated no significant differences when comparing women who received the intervention with their main partners versus women who received the intervention alone. Thus, the intervention does not appear to be any more or less efficacious when a woman received the intervention with her partner compared to when she received it without him. These findings are consistent with the threemonth follow-up when reports of both women and their partners were used in the analysis (El-Bassel, Gilbert et al., 2003). Compared to the findings of ITT analysis, we found that the completers analysis yielded stronger treatment effects.

The strength of these findings is that behavior change was demonstrated at 12 months postintervention, and therefore, maintained over time. These findings are critical in light of the dearth of relationship-based or couple-level HIV/STI risk reduction interventions demonstrating sustained risk reduction outcomes over time.

Two factors may explain the lack of divergence in outcomes between the two active conditions. First, the content of the sessions in both active conditions focused on the intimate relationship between the woman and her partner. The relationship context was given primary emphasis even when the women received the intervention without her partner. This "relationship-based" or more mutual approach to risk reduction has not been included in most HIV/STI prevention interventions for women. All exercises in each session and homework assignments were geared toward working with the recruited study partners. In both active conditions, the women were asked to practice communication, negotiation and condom skills that they learned in the sessions with this partner. The intervention enabled women and their partners to discuss sexual issues and explore together how they can protect themselves from HIV/STIs. Second, the selection criteria of recruiting women with male partners who would be interested and willing to participate in the study may have increased the likelihood that these partners would be receptive to their female partners' attempts to initiate and sustain safer sex practices.
In addition to testing the long-term efficacy of this relationship-based HIV/STI intervention, this paper builds on short-term outcome findings by considering the effect of having completed the intervention (attended all six intervention sessions). For all comparisons involving the interventions versus the education (i.e., couple and woman-alone vs. education, couple versus education, woman alone vs. education), the treatment effect estimates were generally more beneficial when using only completers, compared to estimates derived from completers and non-completers (i.e., ITT). Given that care was taken to ensure appropriate comparisons were made with completers and matched counterparts in the education control condition, the findings provide additional evidence, suggesting that the relationship-based HIV/STI intervention is efficacious in long-term reduction of sexual risk behavior.

The limitations of this study should be considered. The relatively small sample size and selfselection of more "motivated" couples limit our ability to generalize based on these findings. The difference in attrition rates between women assigned to the two active intervention conditions and women assigned the education control condition may have biased study findings despite the use of intention-to-treat approach to minimize potential bias in treatment effect estimates. Another critique of this study may be the lack of biological outcomes on STIs. We had planned to include reports of new STI diagnoses at follow-up as an outcome; however, the incidence of biologically confirmed STIs was extremely low in another study with our study population (NIMH Multisite HIV Prevention Trial, 1998). Continued testing of this intervention with larger sample sizes, both partners, and inclusion of biological markers of treatment outcomes, such as incidence of STIs, is important. In addition, further research is needed to examine how mediators such as communication and negotiation skills, problem solving and condom self-efficacy affect outcomes.

Despite these limitations, the results of the study lend support to the desirability of delivering relationship-based HIV/STI prevention interventions in primary care settings for women and their intimate partners who are at elevated risk for transmission of HIV and other STIs. The results also support the notion that risk reduction behavior can be maintained over time. Finally, the findings have considerable public health implications because they provide evidence of sustained efficacy of a relationship-based intervention for reducing HIV risk behavior in two alternative 
modalities over a 12-month post-intervention time period.

\section{ACKNOWLEDGMENTS}

This study was supported by NIMH Grant MH57145 awarded to Dr El-Bassel.

\section{REFERENCES}

Ajzen, I., and Fishbein, M. (1980). Understanding attitudes and predicting social behavior. Englewood Cliffs, New Jersey: Prentice-Hall.

Allen, S., Serufilira, A., Bogaerts, J., Van de Perre, P., Nsengumuremyi, F., Lindan, C., Carael, M., Wolf, W., Coate, T., and Hulley, S. (1992). Confidential HIV testing and condom promotion in Africa: Impact on HIV and gonorrhea rates. JAMA, 268(23), 3338-3343.

Bandura, A. (1986). Social foundations of thought and action: A social and cognitive theory. Englewood Cliffs, New Jersey: Prentice-Hall.

Bandura, A. (1994). Social cognitive theory and exercise of control over HIV infection. In R. J. DiClemente (Ed.), Preventing AIDS: Theories and methods of behavioral interventions (pp. 25-29). New York: Plenum Press.

Baugh, C. W., Avery, A. W., and Sheets-Haworth, K. L. (1982). Marital Problem-Solving Scale: A measure to assess relationship conflict negotiation ability. Family Therapy, 9(1), 4351.

Becker, M. H. (1974). The Health Belief Model and personal health behavior. Health Education Monograph, 2, 324-473.

Bronfenbrenner, U. (1979). The ecology of human development. American Psychologist, 32, 513-531.

Bryan, A. D., Aiken, L. S., and West, S. G. (1996). Increasing condom use: Evaluation of a theory-based intervention to prevent sexually transmitted diseases in young women. Health Psychology, 15, 371-382.

Catania, J. A., Kegeles, S. M., and Coates, T. J. (1990). Towards an understanding of risk behavior: An AIDS Risk Reduction Model (ARRM). Health Education Quarterly, 17, 53-72.

Centers for Disease Control and Prevention. (2001). HIV/AIDS Surveillance Supplemental Report. Atlanta, GA: US Department of Health and Human Services.

Dehejia, R., and Sadek, W. (1999). Causal effects in nonexperimental studies: Re-evaluating the evaluation of training programs. Journal of the American Statistical Association, 94, 1053-1062.

Deschamps, M., Pape, J., Haffner, A., Hyppolite, R., and Johnson, W. (1991). Heterosexual activity in at risk couples for HIV infection. Paper presented at the the VII International Conference on AIDS, Florence, Italy.

Ehrhardt, A. A., and Exner, T. (2000). Prevention of sexual risk behavior for HIV infection with women. AIDS, 14(2), S53S58.

Ehrhardt, A. A., Yingling, S., Zawadzki, R., and Martinez-Ramirez, M. (1992). Prevention of heterosexual transmission of HIV: Barriers for women. Journal of Psychology \& Human Sexuality, 5, 37-67.

El-Bassel, N., and Schilling, R. F. (1992). 15 month follow-up of women methadone patients taught to reduce heterosexual HIV transmission. Public Health Reports, 107, 500-503.

El-Bassel, N., Witte, S. S., Gilbert, L., Sormanti, M., Moreno, C., Pereira, L., Elam, E., and Steinglass, P. (2001). HIV preven- tion for intimate couples: A relationship-based model. Families, Systems, and Health, 19(4), 379-395.

El-Bassel, N., Witte, S. S., Gilbert, L., Wu, E., Chang, M., Hill, J., and Steinglass, P. (2003). The efficacy of a relationshipbased HIV/STD prevention program for heterosexual couples. American Journal of Public Health, 93(6), 963-969.

Fagan, J. A., and Wexler, S. (1987). Crime at home and in the streets. Violence and Victims, 2(1), 5-23.

Fishbein, M., and Middlestadt, S. E. (1989). Using the theory of reasoned action as a framework for understanding and changing AIDS-related behaviors. In J. N. Wasserheit (Ed.), Primary prevention of AIDS: Psychological approaches (pp. 92-110). Newbury Park, NJ: Sage Publications.

Gilbert, L., El-Bassel, N., Schilling, R., Wada, T., and Bennet, B. (2000). Partner violence and sexual HIV risk behaviors among women in methadone treatment. AIDS and Behavior, 4(3), 261-269.

Harlow, L., Quina, K., Morokoff, P., Rose, J., and Grimley, D. (1993). HIV risk in women: A multifaceted model. Journal of Applied Biobehavioral Research, 1, 3-38.

Harvey, S. (2000). New kinds of data, new options for HIV prevention among women: A public health challenge. Health Education and Behavior, 27(5), 539-565.

Hendrick, C., Hendrick, S., and Dicke, A. (1998). The Love Attitudes Scale: Short form. Journal of Social and Personal Relationships, 15, 147-159.

Higgins, D. L., Galavotti, C., O’Reilly, K. R., Schnell, D. J., Moore, M. M., Rugg, D. L., and Johnson, R. (1991). Evidence for the effects of HIV antibody counseling and testing on risk behaviors. JAMA, 266, 2419-2429.

Ickovics, J. R., and Yoshikawa, H. (1998). Preventive interventions to reduce heterosexual HIV risk for women: Current perspectives, future directions. [Review]. AIDS, 12(Suppl. A), S197S208.

Janz, N. K., and Becker, M. H. (1984). The Health Belief Model: A decade later. Health Education Quarterly, 11, 1-47.

Kalichman, S. C., Kelly, J. A., Hunter, T. L., Murphy, D. A., and Tyler, R. (1993). Culturally-tailored HIV/AIDS risk reduction messages targeted to African American urban women: Impact on risk sensitization and risk reduction. Journal of Consulting and Clinical Psychology, 61, 291-295.

Kalichman, S. C., Williams, E. A., Cherry, C., Belcher, L., and Nachimson, D. (1998). Sexual coercion, domestic violence, and negotiating condom use among low-income African American women. Journal of Women's Health, 7, 371-378.

Kamb, M., Fishbein, M., Douglas, J., Rhodes, F., Rogers, J., Bolan, G., Zenilman, J., Hoxworth, T., Malotte, C., Iatesta, M., Kent, C., Lentz, A., Graziano, S., Byers, R., and Peterman, T. (1998). Efficacy of risk-reduction counseling to prevent human immunodeficiency virus and sexually transmitted diseases: A randomized controlled trial. JAMA, 280(13), 1161-1167.

Landis, S. E., Schoenback, V., and Weber, D. (1992). Results of a randomized trial of partner notification in cases of HIV infection in North Carolina. New England Journal of Medicine, 326, 101-106.

Marin, B. V., Gomez, C. A., Tschann, J. M., and Gregorich, S. E. (1997). Condom use in unmarried Latino men: A test of cultural constructs. Health Psychology, 15(5), 458-467.

McCusker, J., Goldstein, R., Bigelow, C., and Zorn, M. (1993, June 6-11). HIV risk reduction and psychiatric diagnosis among drug users. Paper presented at the IX International Conference on AIDS/HIV STD World Congress, Berlin, Germany.

Misovich, S. J., Fisher, J. D., and Fisher, W. A. (1997). Close relationships and elevated HIV risk behavior: Evidence and possible underlying psychological processes. Review of General Psychology, 1(1), 72-107.

Moss, H. B., and Tarter, R. E. (1993). Substance abuse, aggression and violence: What are the connections? American Journal on Addictions, 2(2), 149-159. 
Musaba, E., Morrison, C. S., Sunkutu, M. R., and Wong, E. L. (1998). Long-term use of the female condom among couples at high risk of human immunodeficiency virus infection in Zambia. Sexually Transmitted Diseases, 25(5), 260-264.

NIMH Multisite HIV Prevention Trial. (1998). Reducing sexual risk behavior. Science, 280, 1889-1894.

O'Leary, A. (2000). Women at risk for HIV from a primary partner: Balancing risk and intimacy. Annual Review of Sex Research, $11,191-234$

Padian, N. S., O'Brien, T. R., Chang, Y. C., Glass, S., and Francis, D. (1993). Prevention of heterosexual transmission of human immunodeficiency virus through couple counseling. Journal of Acquired Immune Deficiency Syndromes, 6, 10431048.

Remien, R. (1997). Couples of mixed HIV status: Challenges and strategies for intervention with couples. In L. Wicks (Ed.), Psychotherapy and AIDS (pp. 165-177). Washington, DC: Taylor \& Francis.

Reynolds, J. (1993). Feminist theory and strategy in social work. In J. Walmsley, J. Reynolds, P. Shakespeare \& R. Woolfe (Eds.), Health welfare and practice: Reflecting on roles and relationships (pp. 74-82). London: Sage Publications.

Rojek, C., Peacock, G., and Collins, S. (1989). Social work and received ideas. London: Routledge Press.

Rosenbaum, P. R., and Rubin, D. B. (1983). The central role of the propensity score in observational studies for causal effects. Biometrika, 70, 41-55.

Rosenbaum, P. R., and Rubin, D. B. (1985). Constructing a control group using multivariate matched sampling methods that incorporate the propensity score. The American Statistician, 39, 33-38.

Rubin, D. B. (1987). Multiple imputation for nonresponse in surveys. New York: Wiley Publications.

Schiff, M., Witte, S., and El-Bassel, N. (2003). Client satisfaction and perceived helping aspects of an HIV/AIDS preventative intervention for urban couples. Research on Social Work Practice, 13(4), 468-492.
Schilling, R. F., El-Bassel, N., Hadden, B., and Gilbert, L. (1995). Skills-training groups to reduce HIV transmission and drug use among methadone patients. Social Work, 40(1), 91101

Shain, R. N., Piper, J. M., Newton, E. R., Perdue, S. T., Ramos, R., Champion, J. D., and Guerra, F. A. (1999). A randomized, controlled trial of a behavioral intervention to prevent sexually transmitted disease among minority women. New England Journal of Medicine, 340(2), 93-100.

Sormanti, M., Pereira, L., El-Bassel, N., Witte, S., and Gilbert, L. (2001). The role of community consultants in designing an HIV prevention intervention. AIDS Education and Prevention, 13(4), 311-328.

Straus, M., Hamby, S., Boney-McCoy, S., and Sugarman, D. (1996). The Revised Conflict Tactics Scales (CTS2): Development \& preliminary psychometric data. Journal of Family Issues, 17, 283-316.

Voluntary HIV-1 Counseling and Testing Efficacy Study Group. (2000). Efficacy of voluntary HIV-1 counselling and testing in individuals and couples in Kenya, Tanzania and Trinidad: A randomized trial. The Lancet, 356, 103-112.

Weiner-Davis, M. (1998). It takes one to tango. Family Therapy Networker (Sept/Oct), 46-51.

Wingood, G. M., and DiClemente, R. J. (1998). Partner influences and gender-related factors associated with noncondom use among young adult African American women. American Journal of Community Psychology, 26(1), 2951.

Wingood, G. M., and DiClemente, R. J. (2000). Application of the theory of gender and power to examine HIV-related exposures, risk factors, and effective interventions for women. Health Education \& Behavior, 27(5), 539-565.

Wu, E., El-Bassel, N., Witte, S., Gilbert, L., and Chang, M. (2001). Correlates of participation in a relationship-based HIV/STD prevention trial. Paper presented at the NIMH Conference on the Role of Families in Preventing and Adapting to HIV/AIDS, Los Angeles, CA. 